\title{
Structure and Dynamics of Ionospheric Plasma
}

\author{
Lucilla Alfonsi ${ }^{1}$ and Libo Liu ${ }^{2}$ \\ ${ }^{1}$ Istituto Nazionale di Geofisica e Vulcanologia, Via di Vigna Murata 605, 00143 Rome, Italy \\ ${ }^{2}$ Institute of Geology and Geophysics, Chinese Academy of Sciences, Beijing 100029, China
}

Correspondence should be addressed to Lucilla Alfonsi, lucilla.alfonsi@ingv.it

Received 29 September 2011; Accepted 29 September 2011

Copyright (C) 2011 L. Alfonsi and L. Liu. This is an open access article distributed under the Creative Commons Attribution License, which permits unrestricted use, distribution, and reproduction in any medium, provided the original work is properly cited.

This special issue is dedicated to the memory of Dr. Olga Pirog, who was the actual Guest Editor. The obituary below, prepared by Nelya Polekh on behalf of all her colleagues of the Irkutsk Scientific Center (Russian Academy of Sciences), opens the issue recalling to our readers her excellence and her enthusiasm as scientist and woman.

Olga Pirog was born in 1941 in Kirensk (Irkutsk region). After finishing the Irkutsk State University, she worked in Norilsk. This determined the areas of her scientific interests: high-latitude ionosphere and the magnetosphere-ionosphere interaction.

In 1974, she successfully defended her thesis for the degree of doctor of physical and mathematical sciences. She was the author of more than 150 scientific articles published in Russian and foreign journals.

Olga Pirog was a highly qualified expert in the physics of the ionosphere and the magnetosphere-ionosphere interaction. The main directions of her research were related to the experimental study of large-scale high-latitude ionosphere structures and their dynamic regime, the main ionospheric trough, manifestations of magnetospheric substorms in the ionosphere disturbances, and study of the response of the ionosphere to geomagnetic storms. Her scientific achievements in the physics and morphology of high- and midlatitude ionosphere and ionospheric-magnetospheric relationships are well known in Russia and abroad and were presented at National conferences and International Symposiums.

She loved traveling and tourism and was interested in literature, music, painting, and history. She loved life. The tragic death happened on December, 16, 2010, crossed her plans. We lost a nice colleague, a good partner, and the beautiful woman. We will always cherish those unforgettable memories of her.

Even if nowadays the ionospheric plasma can be probed by a variety of new technologies, such as LEO satellites, rockets, incoherent scatter radars, backscattering radars, and GNSS receivers, the understanding of its physical properties is still matter of scientific debate. Its structure can change significantly in space, giving rise to the formation of electron density irregularities of different scale sizes, from thousands of kilometers to few centimeters. Ionospheric plasma shows a high degree of variability in time confirmed by a complex dynamics observed over time scales from milliseconds to decades and more. The understanding of the configuration of the ionospheric plasma is not only a scientific challenge, but can also address the needs of our society, focusing on phenomena that can significantly impair a wide range of systems and applications that are at the core of several activities embedded in our daily life. In this direction, space-weather issues, dealing with the harmful effects of the ionosphere on technological systems, will become increasingly significant as we approach the next solar maximum, predicted for 2013.

The selection of the topics included in this issue is far from being exhaustive, but it contributes to demonstrate how many questions arise from the investigation of the coupling between ionized and neutral atmosphere and from the solarterrestrial relationship. We would like to thank the authors for their excellent contributions and patience in assisting us. Finally, the fundamental work of all reviewers on these papers is also very warmly acknowledged.

This special issue contains five papers: two of them investigate the plasma irregularities from observations and from numerical simulation; one deals with the effects of solar wind 
pressure in the high latitude ionosphere in both hemispheres; another paper reports the investigation of the connection between the ionosphere and the underlying atmosphere; finally, the last paper is an overview of the ionosphereatmosphere coupling. The first paper is by Huang, titled "Occurrence of equatorial plasma bubbles during intense magnetic storms" and presents the measurements of the ion density and velocity in the evening equatorial ionosphere by the Defense Meteorological Satellite Program (DMSP) satellites during 22 intense magnetic storms. The results of this study provide evidence that penetration electric field associated with southward IMF during the main phase of magnetic storms increases the generation of equatorial plasma bubbles in the evening sector.

The second paper, by O. V. Mingalev et al., "Numerical simulation of the time evolution of small-scale irregularities in the F-layer ionospheric plasma," addresses the study of the dynamics of magnetic field-aligned small-scale irregularities in the electron concentration, existing in the F-layer ionospheric plasma, investigated with the help of a mathematical model. The results of simulation indicate that the smallscale irregularity, created initially in the F-region ionosphere, decays accomplishing periodic damped vibrations, with the process being collisionless.

In the third paper, "Effects of abrupt variations of solar wind dynamic pressure on the high-latitude ionosphere," I. Coco and coauthors show the results of a statistical study on the effects in the high-latitude ionosphere of abrupt variations of solar wind dynamic pressure, using Super Dual Auroral Radar Network (SuperDARN) data in both hemispheres. Their results show that, during periods of quiet ionospheric conditions, the amount of radar backscatter increases when a variation in the dynamic pressure occurs, both positive (increase of the pressure) and negative (decrease of the pressure).

The fourth paper, "The Planetary wave activity in temperatures of the stratosphere, mesosphere, and in critical frequencies of ionospheric F2 layer," by N. M. Polekh et al., reports the analysis of vertical-incidence ionospheric sounding data and temperature measurements at stratospheric $(30 \mathrm{~km})$ and mesospheric $(80 \mathrm{~km})$ heights to investigate the interrelation between the occurrence of fluctuations with periods of planetary waves in temperature variations at different heights and in F2 layer critical frequency variations (foF2) under low solar activity conditions for the time interval 2006-2007. Their findings reveal a similarity between periodic structures for the variations in the parameters involved.

In the last paper, "Electrodynamical coupling of earth's atmosphere and ionosphere: an overview," A. K. Singh et al. give a brief review of the electrical processes coupling the atmosphere and ionosphere, reporting their present understanding of how these events play key role in energy transfer from the lower atmosphere to the ionosphere.

Lucilla Alfonsi

Libo Liu 

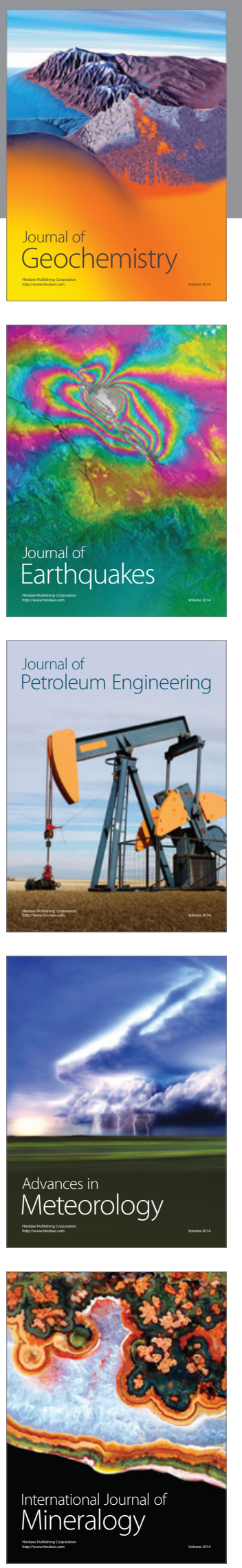
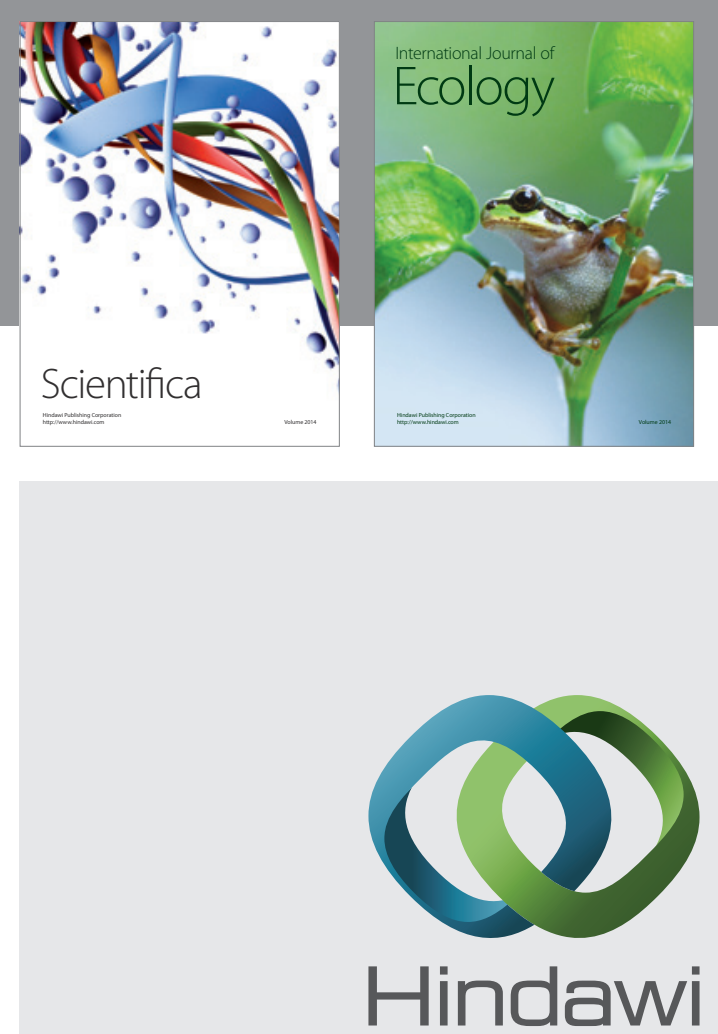

Submit your manuscripts at http://www.hindawi.com
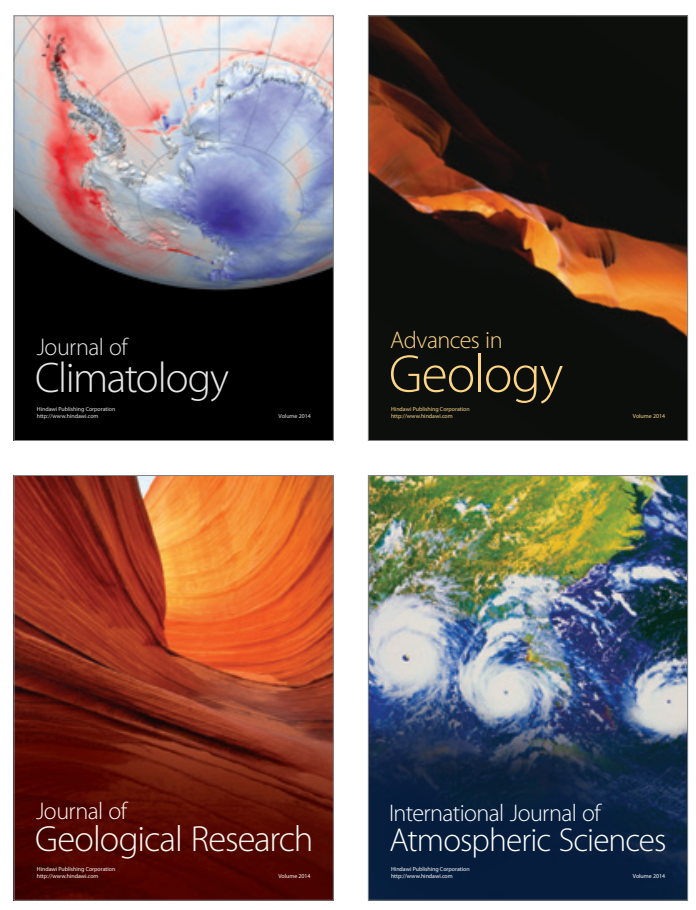
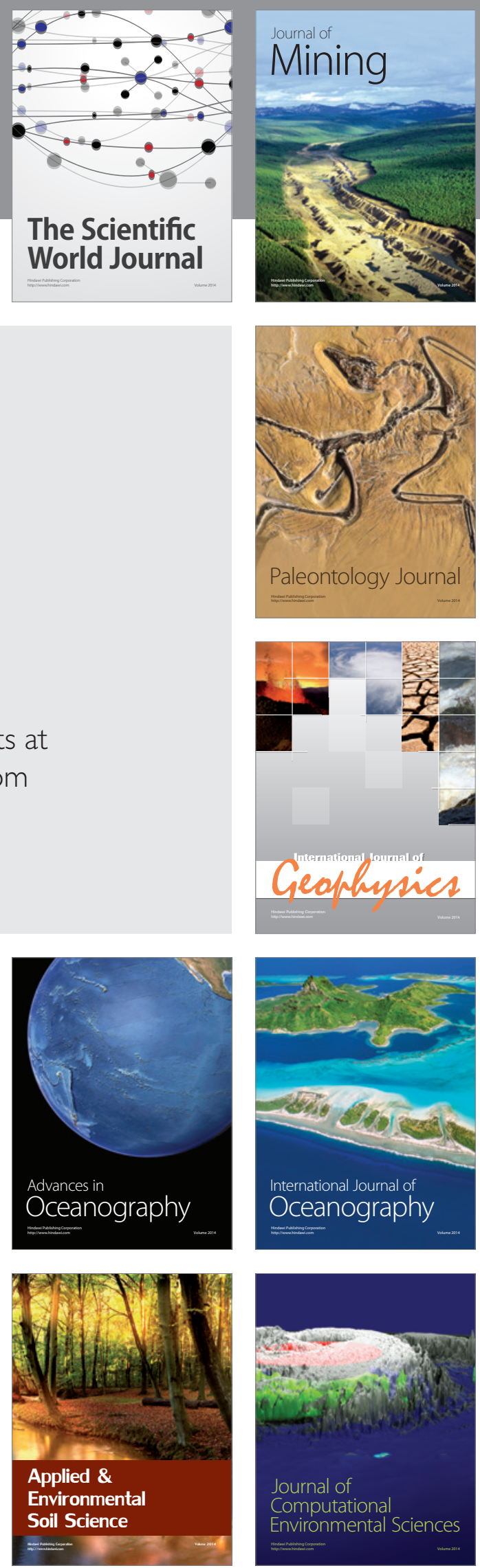